\title{
Simulation Study of Topological Point Defects in Graphitic Layer - Curvature Effect and Pair Correlation Function Analysis
}

\author{
L. HAWELEK* \\ Institute of Non-Ferrous Metals, J. Sowińskiego 5, 44-100 Gliwice, Poland \\ (Received June 6, 2016; in final form July 22, 2016)
}

\begin{abstract}
The effect of three types of topological defects, single vacancy, double vacancy and the Stone-Thrower-Wales defect on the atomic arrangement in a single graphitic layer is studied using computer simulations. The topological defects were positioned on the perfect hexagonal graphitic layer $20 \AA$ in diameter with different distance from the layer edge and then the geometry of the system was independently optimized using the reactive bond order potential, the semi-empirical quantum-chemical PM7 and the density functional theory method. Curvature and the distortion of the graphitic layer caused by the defects are analyzed and their influence on the pair correlation function is discussed.
\end{abstract}

DOI: $10.12693 /$ APhysPolA.130.811

PACS/topics: 81.05.ue, 61.72.J-, 61.43.Bn, 61.66.Fn, 61.05.C-

\section{Introduction}

The structure of most of known carbon nanomaterials such as: carbon nanotubes [1], carbon nanohorns [2], carbon nanoonions [3], activated carbons [4], nanographene platelets [5], and the group of the carbonaceous materials defined as porous carbons [6] may be reconstructed by rolling up of a single graphitic layer or building up as an arrangement of planar single- and multi-layer graphiticlike fragments. Such materials are obtained by various physicochemical processes and possess outstanding electronic, optical, mechanical and absorbing properties directly connected with their atomic scale structure. Some of these properties can be dramatically altered by the presence of structural disorder in the form of defects that may be formed during the synthesis stage. Defects change the local charge distribution in the hexagonal lattice hence change the electron system of $s p^{2}$-bonded carbon materials. Modified bonds around defects affect also the thermal conductivity and the mechanical strength. The defects that exist in nanomaterials are generally divided into extrinsic defects in the form of foreign atoms and intrinsic defects as zero- and one-dimensional defects. The most popular defects are zero-dimensional defects (also named point defects): the Stone-ThrowerWales (STW), single vacancy (SV) and double vacancy (DV) $[7,8]$. The most energetically favorable and stable is the STW defect with its formation energy of $5 \mathrm{eV}[9]$. During formation of this defect the four hexagons are transformed into two pentagons and two heptagons by rotating one of the $\mathrm{C}-\mathrm{C}$ bonds by $90^{\circ}$. The point defect referred as SV is created when one atom is missing from the lattice and the pair of five- and nine-membered rings are

*e-mail: lukasz.hawelek@imn.gliwice.pl formed with the formation energy higher than for STW defects and equals about $7.5 \mathrm{eV}[10,11]$. The last mentioned defect - DV - has similar energy formation as SV (about $8 \mathrm{eV}$ ) and instead of four perfect hexagons the pair of pentagons and octagon appears in the distorted layer when two carbon atoms are missing. As it was shown in our previous studies on various carbonaceous materials by combining the X-ray and neutron diffraction method with different simulation methods and theoretical approaches the nonhexagonal rings may introduce positive/negative curvature in the atomic arrangement $[1-6]$. Such changes in layer curvature have significant influence on average atomic distance distribution well visualized in the real space representation as a pair correlation function $(\mathrm{PCF})$. $\mathrm{PCF}$ is a well-known function usually used in analysis of non-periodic structures.

Structural information about various carbon nanomaterials used to be obtained from studies based on direct imaging methods, such as high resolution transmission electron microscopy or scanning probe microscopy. But these techniques can be regarded as local probes providing information for a small sample volume. Although these methods give valuable information about materials allowing obtaining their structural characterization, a complementary technique for probing a larger sample volume is required. Neutron and X-ray diffraction can be used for that purpose. Interpretation of the diffraction data in real space is based on the pair correlation function analysis for which modelling studies are crucial. Model based simulations can be compared with the experimental data and to validate the model.

In this paper it is demonstrated that the curvature of graphitic layer fragment strongly depends on the presence of the topological point defects. I will particularly focus on the influence of zero-dimensional defects such as the Stone-Thrower-Wales defect, single- and double vacancies on structural disorder of relaxed atomistic models 
using computer simulation methods with various theory level. The disorder will be identified in the real space in the form of the pair distribution function. Computer simulations will focus on geometrical relaxation of the disordered structure to minimize its energy using classical energy minimization with the reactive bond order potential, semi-empirical and density functional theory method. Finally, the curvature effect of various point defects will be precisely described.

\section{Computational methods}

First of all, the atomic models were defined by sets of Cartesian coordinates of the single graphitic layer with size of $20 \AA$ in diameter and generated single point defect such as SV, DV, and STW in three various positions according to the description in Fig. 1. As it is shown in this figure the SV1, DV1, and STW1 defects are placed in the layer center, the SV2, DV2, STW2 defects are placed in the next neighboring positions and SV3, DV3, STW3 are in the closest to the edge positions. For saturation of the dangling bonds, hydrogen atoms were added at the edge of the layer. Then, the energy of such constructed models was minimized using classical molecular dynamics (MD), semi-emprical method (S-EM) and density functional theory (DFT) in order to obtain stable atomic configurations. In the classical molecular dynamics, interactions between carbon atoms are described by the reactive empirical bond order (REBO) potential [12]. It is noteworthy that the REBO potential accounts very well for the features of $s p, s p^{2}$ and $s p^{3}$ bonding, and was especially developed to optimize the geometry of various hydrocarbon and carbon structures. The same initial models of defected graphitic layer were also independently optimized using MOPAC 2012 (Molecular Orbital a)

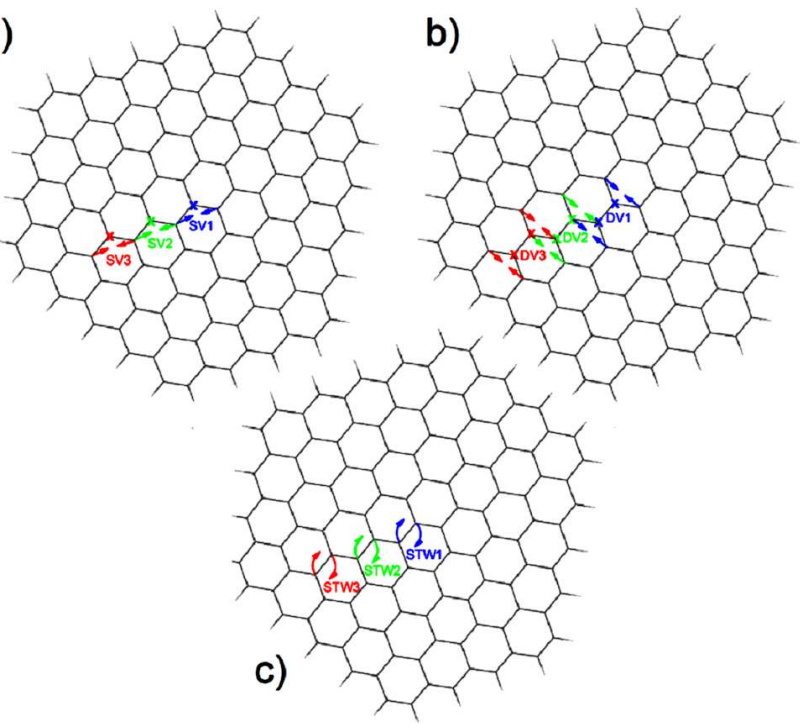

Fig. 1. Position of the topological point defects: single vacancy SV (a), double vacancies DV (b), and the Stone-Thrower-Wales defects STW (c). Crosses mark atoms removed from the layer, arrows define atom shift directions.
Package) with the PM7 semi-empirical quantum chemical method developed by Stewart [13]. Additionally, models relaxation with use of density functional theory at B3LYP $/ 6-31 g(d)$ level using the Orca package were performed [14].

The simulation results were used to calculate the theoretical structure factors and then pair correlation functions only for the carbon atoms of the final configurations. To analyze the interatomic distances of the optimized models in the real space representation, the simulated structure factors were first computed according to the Debye equation

$$
S(K)=1+\frac{1}{N}\left[\sum_{i, j=1}^{N} \frac{\sin \left(K r_{i j}\right)}{K r_{i j}}\right]_{i \neq j},
$$

where $r_{i j}$ is the inter-atomic distance between the $i$-th and $j$-th atom. Then the pair correlation functions (PCF) were computed as the sine Fourier transforms as follows:

$$
\begin{aligned}
& \mathrm{PCF}=\frac{2}{\pi} \int_{0}^{K_{\max }} K[S(K)-1] \sin (K r) \\
& \times \frac{\sin \left(\pi K / K_{\max }\right)}{\pi K / K_{\max }} \mathrm{d} K,
\end{aligned}
$$

where $K_{\max }$ is the maximum value of the scattering vector (in the presented simulations this value was $K_{\max }=$ $20 \AA^{-1}$, and is available for pulsed neutron and reactor sources as well for synchrotron sources even for a laboratory instrument equipped with silver tube emitting $\operatorname{Ag} K_{\alpha}$ radiation). The theoretical functions were calculated under the same conditions as the experimental ones, thus allowing their direct comparison.

\section{Results and discussion}

As it is mentioned in Sect. 1 the formalism of the pair correlation function is used in this paper (Eq. (2)) for the structural analysis of partially disordered layered carbonaceous materials. Such a defined function oscillates around the zero line and its peaks are related to the carbon-carbon distance distributions averaged over a whole model. The PCFs shown in Figs. 2-4 exhibit structure up to about $14 \AA$ and this value is sometimes defined as the coherence length of the PCF for the selected model. Diameter of the single graphitic layer is $20 \AA$ and oscillations of the PCFs disappear about $14 \AA$ due to distortion of the network resulting from the presence of the defects. The first three peaks of the PCFs are related to the carbon-carbon correlations within hexagon which are equivalent to first three closest $\mathrm{C}-\mathrm{C}$ distances in the graphitic layer: about $1.42 \AA$ for nearest-neighbours, about $2.43 \AA$ for every second neighbour atoms and about $2.85 \AA$ for the longest diagonal of hexagon. Because of the highest numbers of the closest and second neighbors the first two peaks have the highest intensities in the PCFs. The next peaks of the PCFs define the carboncarbon distance distributions between the selected atom 
and the atoms from the adjacent hexagons covering the range of the inter-atomic distances from about $3.5 \AA$ up to $5.3 \AA$.

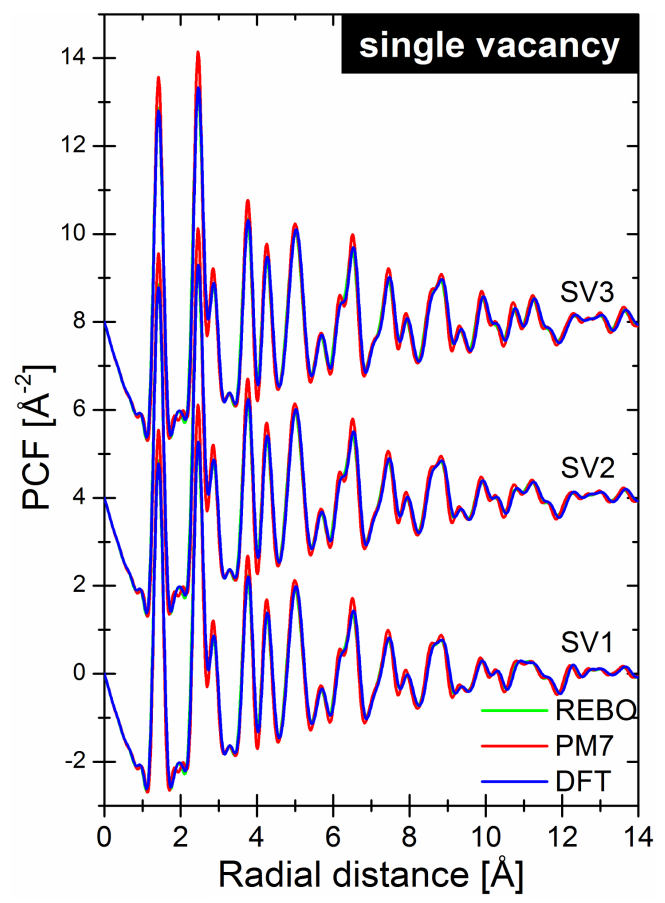

Fig. 2. Comparison of the pair correlation functions calculated from the REBO, PM7, and DFT results for three different defect position MV1, MV2, and MV3 according to Fig. 1a.

Figure 2 illustrates the comparison of the PCFs calculated for models with different single vacancy defect positions SV1, SV2, and SV3, and optimized using the classical molecular dynamics with the REBO potential, PM7, and DFT methods. It is clearly seen that there are almost no discrepancies between positions the PCF peaks for all SV1, SV2, and SV4 defects for the REBO, PM7, and DFT methods. For all three single vacancy defect positions the PCF calculated from the PM7 relaxed models exhibits slightly higher amplitudes when compared with the models relaxed using the REBO and DFT methods. These discrepancies are more pronounced below $8 \AA$. Moreover for the PM7 method slight shifts of the PCF peak positions towards lower values of the inter-atomic distances can be seen in the 8-14 $\AA$ range. On the other hand, the PCFs computed for the DFT and REBO relaxed models practically overlap within the line thickness. The effect mentioned first above is related to much narrower bond distance distribution that causes increase in the amplitude value of the peaks and slightly decreases their full width at half maximum (FWHM) values. The second effect is related to the preservation of the $\mathrm{C}-\mathrm{C}$ distance over the hexagonal net within the relaxation method. In the presence of single vacancy defect the $\mathrm{C}-\mathrm{C}$ distances between the atoms placed close to the opposite layer edge are shorter than for the REBO and
DFT ones. This shortening effect will be proportionally stronger for higher diameters of the graphitic layers. After further careful comparison of the PCFs between the defects SV1, SV2, and SV3, the suppressing effect of the amplitudes positions above $6 \AA$ is clearly seen. The interatomic correlations for this higher distance region are the strongest for SV3 close to layer edge position and are weakened for SV2, and the weakest for SV1 center position of the defect. This effect of the PCF's amplitude dumping is related to coupling of the defect to layer edge distance. The point defect not only disturbs the closest $\mathrm{C}-\mathrm{C}$ bond but also the neighboring hexagons. If the defect is closer to the layer edge, more of the undisturbed hexagons and $\mathrm{C}-\mathrm{C}$ bonds from the other side of the layer give the stronger contribution in the PCFs in higher radial distance values.

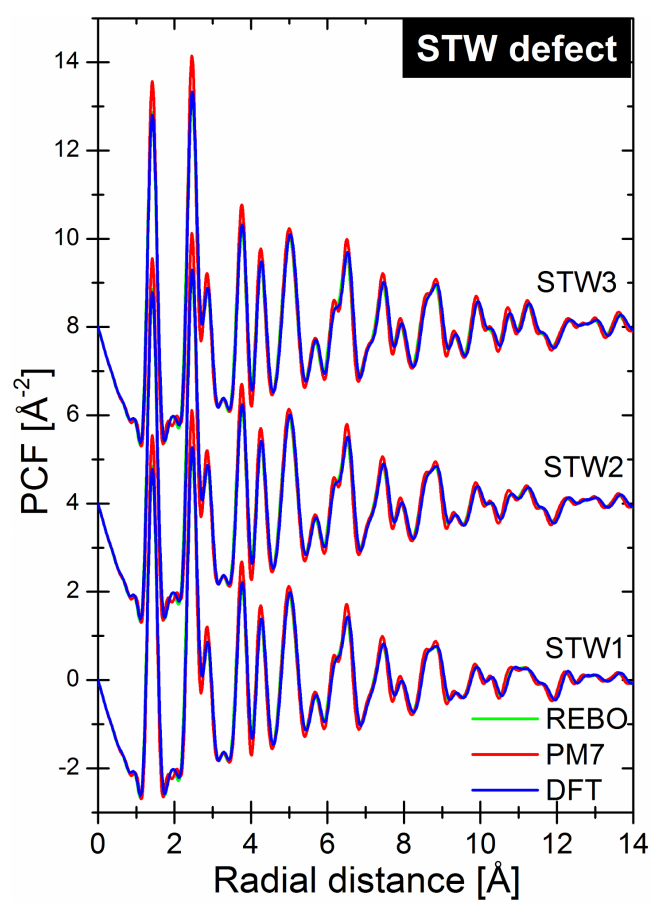

Fig. 3. Comparison of the pair correlation functions calculated from the REBO, PM7, and DFT results for three different defect position STW1, STW2, and STW3 according to Fig. 1c.

Similar interatomic distance analysis of the pair correlation function calculated for the Stone-Thrower-Wales defect in three different positions STW1, STW2, and STW3 was performed. The comparison of the PCFs for the REBO, PM7, and DFT of all three relaxed models is presented in Fig. 3. From the figure inspection it can be easily deduced that similar to SV defects of PM7 method $\mathrm{C}-\mathrm{C}$ bond shortening effect is observed here. As for SV defects this effect may be ignored for atomic cluster up to 5-6 $\AA$ in diameter, and is proportional to interatomic distances. Additionally, in all the three defect positions low-middle radial distance amplitudes have higher maxima and smaller FWHM values. For STW defects the 
effect of amplitude suppression for higher radial distances with different defect position is also observed. For center position of the defect there are more disturbed hexagonal rings in the system than for middle STW2 and close to edge STW3 defect position. It may be clearly depicted from inspection of the PCFs in the range of radial distance in the $10-14 \AA$ range.

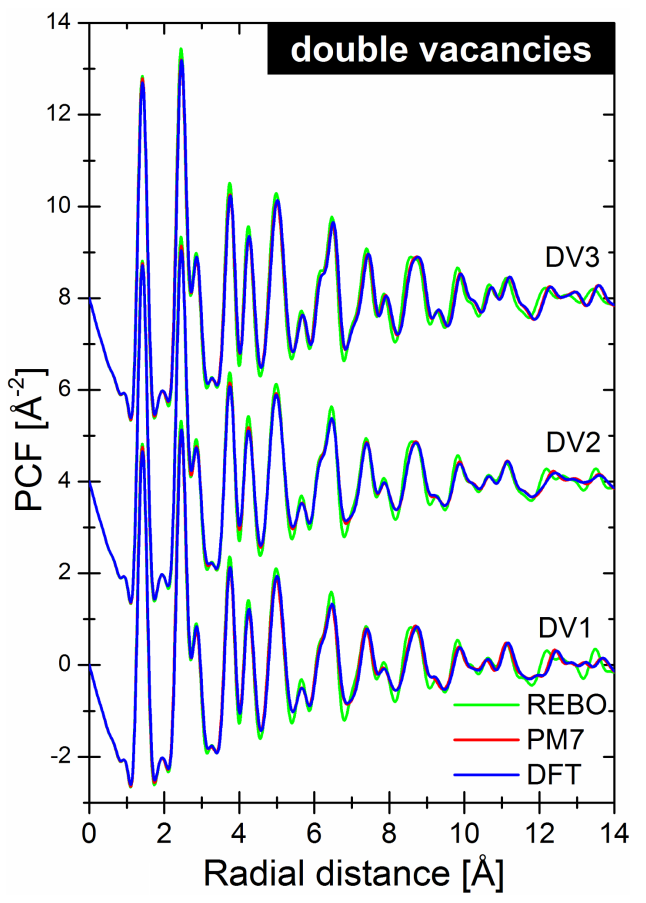

Fig. 4. Comparison of the pair correlation functions calculated from the REBO, PM7, and DFT results for three different defect position DV1, DV2, and DV3 according to Fig. $1 \mathrm{~b}$.

In Fig. 4 the comparison of the PCFs calculated for relaxed models with double vacancies different positions DV1, DV2, and DV3 are gathered. From the first view it is clearly seen that PM7 and DFT methods give almost identical interatomic correlations in the full radial distance range for all three defect position cases, and only very slight shifts of the PM7 peaks maxima into lower values occur. This effect for such model size is almost negligible. However, the PCF calculated for relaxation using the REBO potential possesses some regions in the radial distance that some discrepancies in comparison with the DFT and PM7 optimization methods are clearly seen. For all three defects DV1, DV2, and DV3 two main radial distance ranges of maxima position discrepancies exist: $8-9 \AA$ and $12-14 \AA$. In the first range there is only one broadened peak, but its shape and position is different than obtained from PM7 and DFT relaxation. This peak derives from triple value of $2.75-2.9 \AA$, atoms from the opposite sides in the hexagonal ring, thus this peak defines atomic distance distribution from the opposite sides of every three hexagonal rings. In DV1 and DV2 defect positions in the range $9-12 \AA$ the curves from all three relaxation methods have similar amplitudes, but from $12 \AA$ the REBO relaxed models give shorter $\mathrm{C}-\mathrm{C}$ distances. In the DV 3 position double vacancy defect is in the closest position to the layer edge and the PCF is stronger suppressed than for DV1 and DV2 positions. These inhomogeneous characteristics of the PCFs calculated for the REBO relaxed models in various defect configurations evidently does not come from the systematic difference of the $\mathrm{C}-\mathrm{C}$ bond length between used relaxation methods.

In all three types of point topological defects the nonhexagonal rings give rise to the structural distortion. To verify the differences in $\mathrm{C}-\mathrm{C}$ bond lengths in such distorted structure the average of $\mathrm{C}-\mathrm{C}$ bond lengths inside the pentagons for all defect types in all their configurations have been calculated and gathered in Table I. From the data inspection the PM7 optimization method gives longer average bond in comparison to the DFT and REBO results. That is the only supplementary information on atomic arrangement. To find a more precise answer on the differences in the PCF, a more detailed structural analysis of the layer curvature for the REBO relaxed models has been further performed.

TABLE I

The mean $\mathrm{C}-\mathrm{C}$ bond length value in the pentagons located in the topological defect.

\begin{tabular}{|c|c|c|c|c|c|c|c|c|c|}
\hline \multirow[b]{2}{*}{ Method } & \multicolumn{3}{|c|}{ SV defect $[\AA]$} & \multicolumn{3}{|c|}{ DV defect $[\AA]$} & \multicolumn{3}{|c|}{ STW defect $[\AA]$} \\
\hline & SV1 & SV2 & SV3 & DV1 & DV2 & DV3 & STW1 & STW2 & STW3 \\
\hline PM7 & 1.5029 & 1.5021 & 1.4915 & 1.4583 & 1.4576 & 1.4530 & 1.4383 & 1.4385 & 1.4361 \\
\hline DFT & 1.4982 & 1.4911 & 1.4849 & 1.4541 & 1.4590 & 1.4478 & 1.4222 & 1.4221 & 1.4224 \\
\hline REBO & 1.4952 & 1.4947 & 1.4897 & 1.4514 & 1.4537 & 1.4507 & 1.4322 & 1.4310 & 1.4318 \\
\hline
\end{tabular}

Figures 5, 6 and 7 illustrate the deviation of the $z$-th Cartesian coordination component of the REBO relaxed atomic arrangements of the layer in the presence of topological point defects SV, STW, and DV, respectively.
In all three figures, to simplify the comparison studies, the scale of the $z$-th component is unified, present in the rainbow color scale and the origin of the atomic $z$-th component is equal to 0 . In all the single vacancy positions 
the REBO relaxed structures show symmetrical behavior in regard of 9-5 rings axis. In SV1 and SV2 positions the perpendicular to the axis wings of the layer have relatively positive curvature up to $+1 \AA$ and negative curvature up to about $-1 \AA$ along the symmetry axis. The center of the defect remains in the initial $z$-th component around $0 \AA$. In the close to edge position SV3 the curvature behavior is reverse. The positive curvature is up to about $+0.5 \AA$ along the $9-5$ rings axis and negative curvature perpendicular to the defect axis. However, this negative curvature has asymmetrical shape, the smaller layer wing, which is closer to $9-5$ rings has strong negative curvature with the minimum about $-1.5 \AA$, but the second one, on the opposite site the slope of the layer is gentle with the minimum about $-0.3 \AA$.
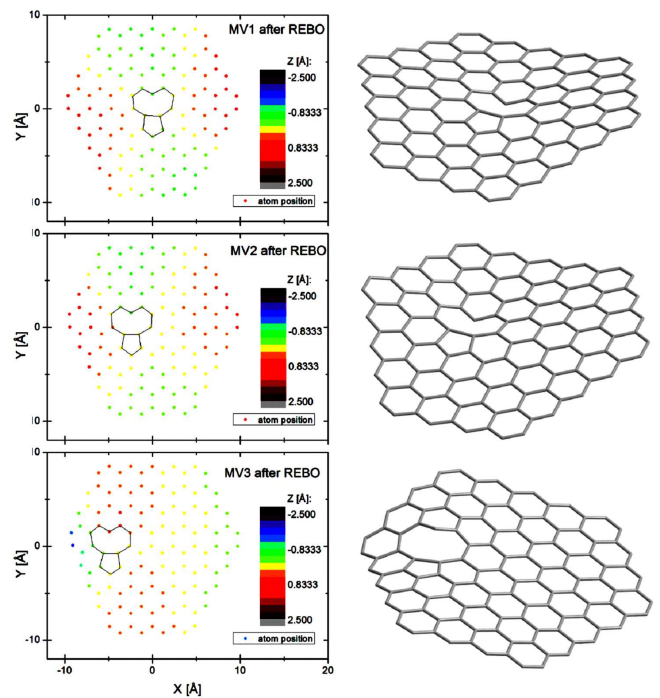

Fig. 5. The curvature effect of single vacancy in three different positions after geometry optimization using REBO potential.

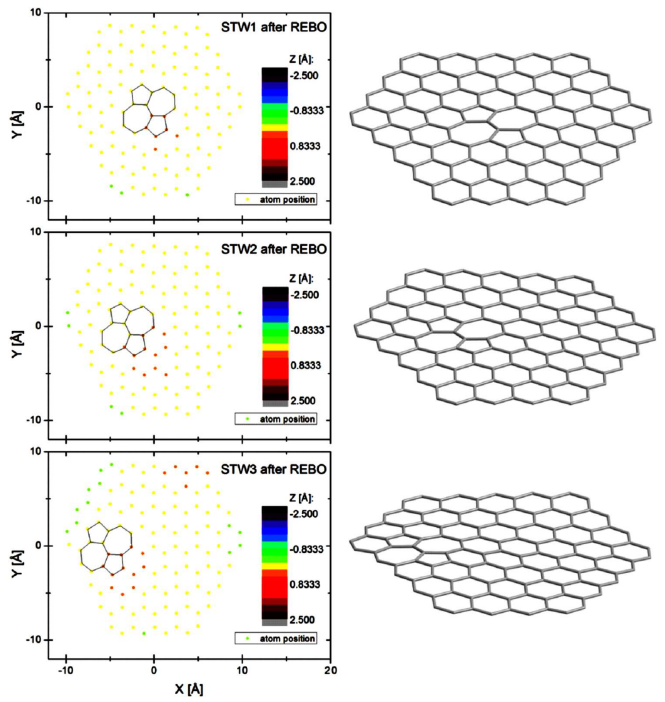

Fig. 6. The curvature effect of Stone-Thrower-Wales in three different positions after geometry optimization using REBO potential.
According to relaxation procedure using the REBO potential the presence of STW defects in their all three positions STW1, STW2, and STW3, the graphene structure remains almost planar (Fig. 6). Only small deviation in the $z$-th component is seen in the edge atom position and in neighboring atoms close to one pentagon of the STW defect. This small positive deviation up to about $+0.3 \AA$ prove the evidence of the most stable sine-like buckled type STW defect presented before in [15].

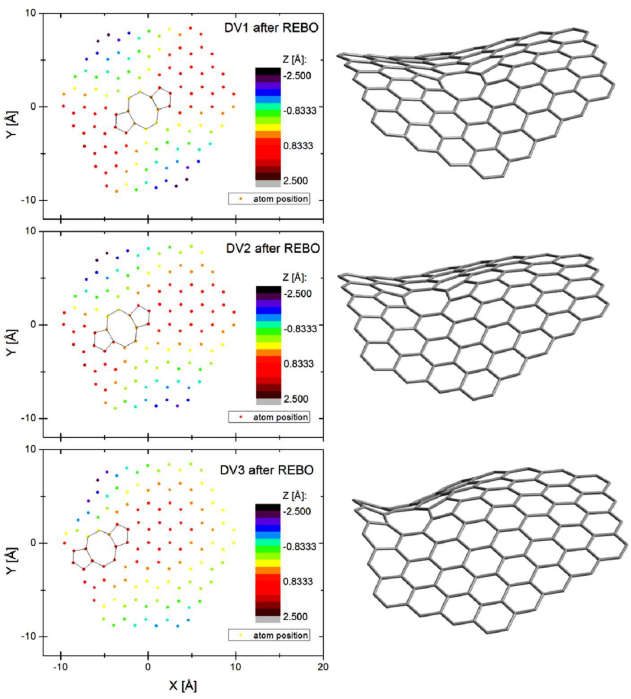

Fig. 7. The curvature effect of double vacancies in three different positions after geometry optimization using REBO potential.

In the case of double vacancy defect, the deviation of $z$ th components, presented in Fig. 7, has similar tendency in all defect positions and the positive curvature is located along the defect axis with the maximum at $+1.5 \AA$ and negative curvature perpendicularly to this axis with the minimum value at the layer edge about $-2.2 \AA$. In all three cases the DV defect generates the similar saddle-like curvature with the maximal difference between the positive and negative deviation from the initial atomic $z$-th component, that equals $3.7 \AA$. This strong $z$-th component deviation gives rise to interatomic distance deviation in middle distance and explains the local shortening distances in the radial distance ranges 8-11 $\AA$ and above $12 \AA$ for REBO relaxed model presented in Fig. 4. This gives the clear evidence of coupling the topological point defect presence with local curvature formation and its influence on the interatomic distances analyzed via the PCF approach.

\section{Conclusion}

The comparative modelling studies of the influence of three most popular topological defects on the local atomic arrangement and graphitic layer curvature showed that the Stone-Thrower-Wales defect gives rise to only slightly sine-like buckled, almost planar structure. 
The presence of single vacancy defect introduces in the REBO relaxed model stronger deviation of atomic $z$-th component up to $\pm 1 \AA$, and the curvature sign correlates with the axis of this topological defect and its distance to the layer edge. The double vacancies defect generate the most distorted structure with the saddle-like curvature and highest $z$-th component deviation, with positive deviation along the defect axis and negative perpendicularly, independently from the distance to the edge position. The pair correlation functions calculated for the REBO relaxed models similarly reconstruct the structural details as the DFT relaxed models in the case of SV and STW defects, for DV defects the REBO relaxation generates more distorted atomic arrangements. The PM7 relaxed models similarly reconstruct the structural details but for less distorted models with STW and SV defects the interatomic distances show some underestimation in the $\mathrm{C}-\mathrm{C}$ bond distances that come from the parametrization method. For more distorted DV defect models this underestimation effect is almost negligible. Thus obtained detailed structural results suggest that the modelling studies of non-periodical, porous and distorted carbonaceous materials should be carefully performed. To reconstruct the atomic arrangement and structural details the knowledge of the both curvature and interatomic distances are of prime importance.

\section{Acknowledgments}

Author would like to thank prof. Andrzej Burian from University of Silesia for fruitful discussion. Author is also grateful for the help from Jacek Hudecki (BielskoBiała, Poland) and Alexandra Maid-Sokol (Edmonton, Canada).

\section{References}

[1] L. Hawelek, J. Koloczek, A. Brodka, J.C. Dore, V. Honkimaki, Y. Ando, A. Burian, Diamond Relat. Mater. 29, 18 (2012).
[2] L. Hawelek, A. Brodka, J.C. Dore, A.C. Hannon, S. Iijima, M. Yudasaka, T. Ohba, K. Kaneko, A. Burian, J. Phys. Chem. A 117, 9057 (2013).

[3] L. Hawelek, A. Brodka, S. Tomita, J.C. Dore, V. Honkimaki, A. Burian, Diamond Relat. Mater. 20, 1333 (2011).

[4] L. Hawelek, A. Brodka, J.C. Dore, V. Honkimaki, A. Burian, J. Phys. Condens. Matter 25, 454203 (2013).

[5] L. Hawelek, A. Kolano-Burian, J. Szade, W. Maziarz, N. Woznica, A. Burian, Diamond Relat. Mater. 35 , 40 (2013).

[6] K. Jurkiewicz, L. Hawelek, K. Balin, J. Szade, F.L. Braghiroli, V. Fierro, A. Celzard, A. Burian, J. Phys. Chem. A 119, 8692 (2015).

[7] F. Banhart, J. Kotakoski, A.V. Krasheninnikov, ACS Nano 5, 26 (2011).

[8] P.A. Thrower, Chem. Phys. Carbon 5, 217 (1969).

[9] L. Li, Phys. Rev. B 72, 184109 (2005).

[10] A.V. Krasheninnkov, P.O. Lehtinen, A.S. Foster, R.M. Nieminen, Chem. Phys. Lett. 418, 132 (2006).

[11] A.A. El-Barbary, R.H. Telling, C.P. Ewels, M.I. Heggie, P.R. Briddon, Phys. Rev. B 68, 144107 (2003).

[12] D.W. Brenner, O.A. Shenderova, J.A. Harrison, S.J. Stuart, B. Ni, S.B. Sinnott, J. Phys. Condens Matter 14, 783 (2002).

[13] J.P.J. Stewart, J. Mol. Model. 19, 1 (2013).

[14] F. Neese, Orca - An ab initio, Density Functional and Semiempirical Program Package, Version 2.5, University of Bonn, 2006.

[15] J. Ma, D. Alf̌́, A. Michaelides, E. Wang, Phys. Rev. B 80, 033407 (2009). 